\title{
Dust cake morphology in fibrous filtration of monodisperse particles based on fractal analysis
}

\author{
Shixian $\mathrm{Wu}^{1, \mathrm{a}}$, Hui Zhu ${ }^{2, \mathrm{~b}}$, Can $\mathrm{Qi}^{3, \mathrm{c}}$, Haiming $\mathrm{Fu}^{4, \mathrm{~d}}$ \\ 1,2,3 Department of Energy Engineering and Building Environment, Guilin University of Aerospace \\ Technology, Guilin 541004, China \\ 4.School of Environmental Science and Engineering, Donghua University, Shanghai 200051, China

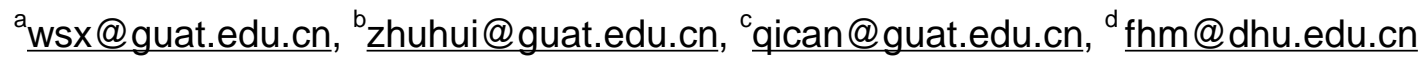

Keywords: fibrous filtration; dust cake; convection-diffusion; fractal dimension

\begin{abstract}
An off-lattice Monte Carlo model has been developed to simulate growth of dust cakes from ultrafine particles in a model filter composed of an array of identical parallel fibers placed transverse to the flow. Unlike previous simulations on the dust cakes, this study considerd the effect of the flow feature in the vicinity of fibers on the morphologies of dust cakes. The flow was described by the modified Kuwabara flow field with consideration of the slip effect at fibers surface. The probability of a particle moving in four different directions was derived from the two-dimensional particle convection-diffusion-migration equation. The resulting microstructure of dust cake formed on fibrous medium was characterized by its density and fractal dimension. The dependences of the porous properties of dust cakes (density, density distribution and fractal dimension) upon the particle transport mechanisms (measured by Pelect number, $P e$ in this work) were also investigated. The results indicate that the dust cakes structures strongly depend on particle transport mechanism, a smaller $P e$ tends to give a more open and loose structure with a lower density and fractal dimension. For the case of $P e=1$, the fractal dimension for the cake structure approaches to 1.710, a value for the diffusion-limited aggregation (DLA) model. When $P e>100$, the deposition behavior is very close to the pure ballistic aggregation model (LA), and the resulting structure is on average uniform at all length scale. Furthermore, a dust layer near filtration medium with lower porosity can be observed for a higher Peclet number $(P e \geq 10)$, unlike previous results on particles deposition on a simply two dimensional threadiness substrate.
\end{abstract}

\section{Introduction}

Fibrous filter are widely applied to filter particulate pollutants from gases because of its ability for collecting micrometric particles with efficiencies above 99\% [1]. Experimental results have clearly shown that the pressure loss across the dust cake during filtration as well as the energy necessary to remove the dust layer in the cleaning cycle strongly depend on cake porosity [2-4].

Nonetheless, knowledge on dynamic behavior for the growth of dust cakes on fibrous medium is still very poor, and predictions of the structure of a dust cake formed during filtration are often difficult to make since the dust cake is very weak and is easily disturbed on handling. A more practical way for obtaining detailed structural information of dust cake is through a computer simulation. The first attempt was made through computer simulations to study the accumulation of solid particles on the surface of a filter medium is that of Houi and Lenormand [5], who simulated the growth of chain-like dendrites on fibers based on Diffusion-Limited-Aggregation (DLA) byWitten and Sander [6]. To investigate the effect of physical parameters of colloidal particles on the morphology of deposits in porous media, Veerapaneni and Wiesner presented an on-lattice Monte Carlo simulation method for simulating colloid deposition on a one-dimensional permeable surface, and dependece of deposition morphology on the relative importance of the deterministic particle motion to the Brownian motion was discussed [7]. Stöcklmayer and Höflinger reported a studies in which the compressible behavior of dust layer composed of monodisperse particles were simulated, in their 
simulations, the dust cake compression was determined by comparing the magnitude of the compression force with that of the resistance force on compression [8]. Later on, Jeon and Jung modified the simulation method to include the effects of the adhesion force between two contacting particles and particle size on the compression behavior of dust cakes, without however considering the Brownian motion of fine particles as well as the flow field across filter media [9].

In this study, as an extension of two dimension on-lattice Monte Carlo simulations method, we developed a off-lattice Monte Carlo simulations method to predict the growth process of the dust cake on a model fibrous filter composed of an array of identical parallel cylinders placed transverse to the flow. The resulting microstructures of the dust cake were described by local densities and fractal dimension, respectively, and the dependences of the cake morphology upon particle transport mechanism were also investigated by using the present simulations method.

\section{Simulation method}

We consider the build-up of dust filter cakes on a two dimensional model filter composed of an array of identical parallel fibers placed transverse to the flow, as shown in Fig. 1. Because of the complexity of the interactions between particles-particles, particles-filtration medium as well as particles-airflow in the real growth process of dust cakes, direct simulation for the real dust cake formation process is still seem to be impossible. A simplified model is therefore needed to be established on basis of the following basic assumptions: (1) The dust cake growth is only governed by combination transport mechanism of particles diffusion and convection, ignoring particle inertia, electrostatic force and impaction rebound upon fiber or previous deposited particles. (2) The particles are spherical and generated at the release surface one at a time using the random number generation program, as shown in Fig. 1. (3) The complex interactions of particle-particle in dust cakes are absent, that is, dust cake is incompressible. (4) The particle deposition behavior is considered for the individual particle in each simulation run. Once the approaching particle makes contact with either fibers or one of deposited particles in dust cake, it is assumed to be deposited at the position of contact. (5) The flow field around the fibers is described in term of the modified Kuwabara flow model as shown in Fig. 1 (right), and the stream line outside of Kuwabara cell is assumed to be rectilinear. (6) The change of the flow field due to the presence of deposits is ignored.

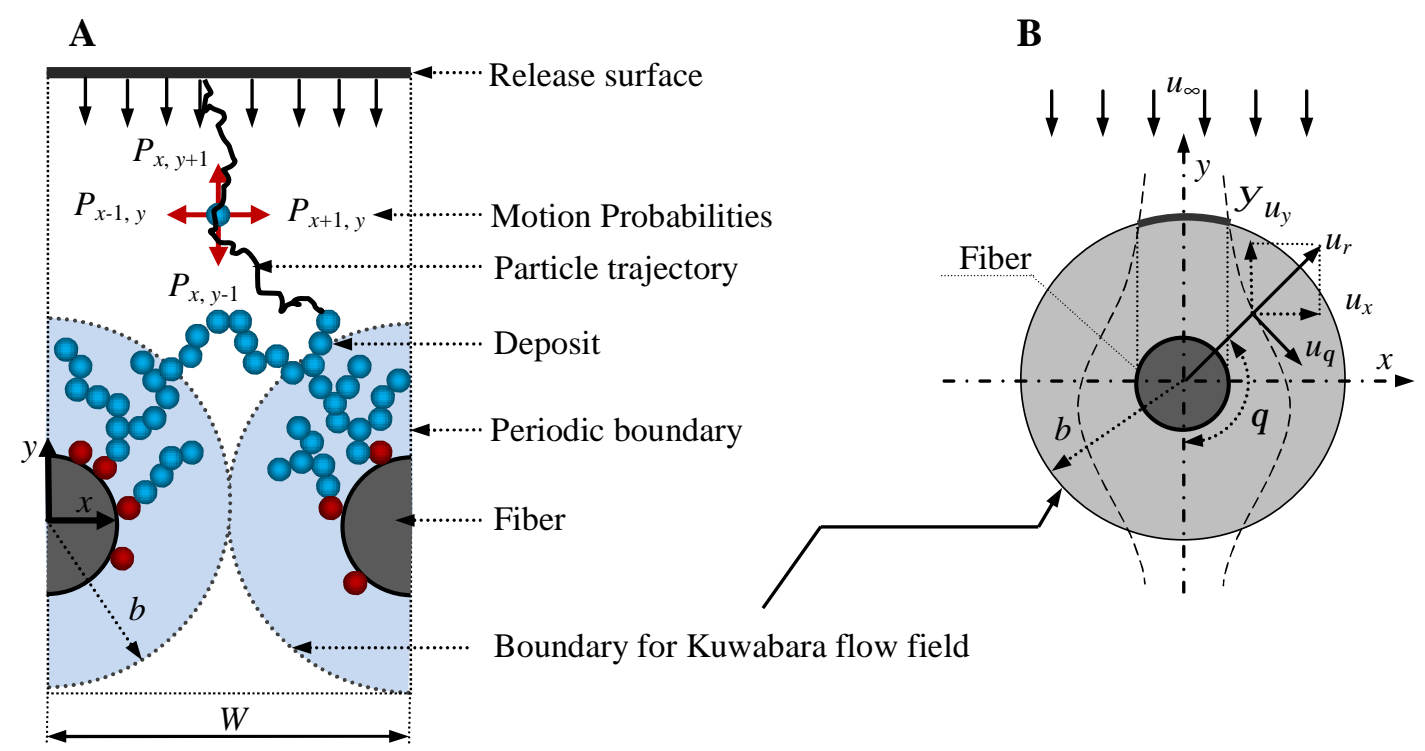

Fig. 1 Schematic of the simulation of dust cake and the Kuwabara flow configuration

Based on the obove physical assumptions, the convection-diffusion equation for the particles within the region of interest is given as: 


$$
D \frac{\partial^{2} n}{\partial x^{2}}+D \frac{\partial^{2} n}{\partial y^{2}}-u_{x} \frac{\partial n}{\partial x}-u_{y} \frac{\partial n}{\partial y}=0
$$

where $D$ is the diffusion coefficient, $n$ is the number of particles per unit volume at position $(x, y)$, and $u_{x}$ and $u_{y}$ are local fluid velocities at $(x, y)$. The first two terms in Eq. 1 are the diffusion terms in the $x$ and $y$ directions, and last two terms are the convection terms in the $x$ and $y$ directions, respectively.

In order to write Eq. (1) in a dimensionless form, the dimensionless variables are introduced as follows

$$
n^{*}=\frac{n}{n_{0}} x^{*}=\frac{x}{l} y^{*}=\frac{y}{l}, u_{x}^{*}=\frac{u_{x}}{u_{\infty}}, u_{y}^{*}=\frac{u_{y}}{u_{\infty}} P e=\frac{u_{\infty} l}{D}
$$

where $n_{0}$ is undisturbed particle concentration, $u_{\infty}$ is undisturbed approach velocity of gas, $l$ is a characteristic length ( $l$ is taken to be unity in the present simulations). By multiplying both sides of Eq. (1) by $l^{2} /\left(D n_{0}\right)$, it may be rewritten in a dimensionless form as

$$
\frac{\partial^{2} n^{*}}{\partial x^{* 2}}+\frac{\partial^{2} n^{*}}{\partial y^{* 2}}-P e\left(u_{x}^{*} \frac{\partial n^{*}}{\partial x^{*}}+u_{y}^{*} \frac{\partial n^{*}}{\partial y^{*}}\right)=0
$$

In the Monter Carlo simulation for growth of dust cake, the particles motion probabilities are derived from the macroscopic conservation equation, i.e., Eq. (2). Now let $\delta x^{*}$ and $\delta y^{*}$ are the step in the $x$ direction and $y$ direction, respectively, then the discrete version of Eq. (2) can be converted by the differential operators as follows

$$
\begin{aligned}
& \frac{\partial n^{*}}{\partial x^{*}}=\left(n_{x, y}^{*}-n_{x-1, y}^{*}\right) / \delta x^{*} \\
& \frac{\partial n^{*}}{\partial y^{*}}=\left(n_{x, y}^{*}-n_{x, y-1}^{*}\right) / \delta y^{*} \\
& \frac{\partial^{2} n^{*}}{\partial x^{* 2}}=\left(n_{x-1, y}^{*}+n_{x+1, y}^{*}-2 n_{x, y}^{*}\right) / \delta x^{* 2} \\
& \frac{\partial^{2} n^{*}}{\partial y^{* 2}}=\left(n_{x, y-1}^{*}+n_{x, y+1}^{*}-2 n_{x, y}^{*}\right) / \delta y^{* 2}
\end{aligned}
$$

Substituting Eqs. (3) - (6) into Eq. (2), and imposing the condition of $\delta x^{*}=\delta y^{*}=1$, we obtain

$$
n_{x, y}^{*}=\frac{n_{x+1, y}^{*}}{4+P e\left(u_{x}^{*}+u_{y}^{*}\right)}+\frac{n_{x-1, y}^{*}\left(1+P e u_{x}^{*}\right)}{4+P e\left(u_{x}^{*}+u_{y}^{*}\right)}+\frac{n_{x, y+1}^{*}}{4+P e\left(u_{x}^{*}+u_{y}^{*}\right)}+\frac{n_{x, y-1}^{*}\left(1+P e u_{y}^{*}\right)}{4+P e\left(u_{x}^{*}+u_{y}^{*}\right)}
$$

Applying the relationship between particles concentration and probability of a particle moving in two dimensional space to Eq. (7) (for details see [10]), the probability of the particle moving in four directions are

$$
P_{x+1, y}=\frac{1}{4+P e\left(u_{x}^{*}+u_{y}^{*}\right)}, P_{x-1, y}=\frac{1+P e u_{x}^{*}}{4+P e\left(u_{x}^{*}+u_{y}^{*}\right)}, P_{x, y+1}=\frac{1}{4+P e\left(u_{x}^{*}+u_{y}^{*}\right)}, P_{x, y-1}=\frac{1+P e u_{y}^{*}}{4+P e\left(u_{x}^{*}+u_{y}^{*}\right)}(8)
$$

As can be seen from the above equation, the motion probabilities depend upon the Peclet number (i.e., $P e$ ) and local gas velocities. Under the limiting condition of $P e \rightarrow 0$, the transport probability of particles in each direction will be $1 / 4$, the deposition behavior reduces to the well known diffusion-limited deposition [11]. On the other hand, for the case of $P e \rightarrow \infty$, the diffusion effect is negligible, and the convection is the dominated transport mechanism. In the middle region, the particles motion is controlled by combination of the particles diffusion and convection. The $P e$ can be therefore used to describe the relative importance of these two particles transport mechanisms (i.e., diffusion and convection). To warrant the value of probability to be non-negative, we take the absolute value of each parameter in Eq. (8).

Note that, in Eq. (8), the determination of the probabilities for particles motion also requires for the knowledge of local flow velocities (i.e., $u_{x}^{*}$ and $u_{y}^{*}$ ) in simulation domain. In the present study,we will restrict the analysis to the filtration at low Reynolds number $(\operatorname{Re}=1)$, so the flow around filtration medium can be considerd to be creeping flow. In such case, the modified Kuwabara flow model 
including the slip effect at fiber surface is valid, and can be used to estimate the flow velocity in the proximity of a fiber. As for the flow field beyond Kuwabara cell, it is assumed to be rectilinear for simplicity. In the polar coordinates in Fig. 1(right), the tream function $\Psi$ for Kuwabara model is described by $[12,13]$

$$
\psi=\frac{2 u r}{J}\left[A\left(\frac{r_{\mathrm{f}}}{r}\right)^{2}+B+C \ln \left(\frac{r_{\mathrm{f}}}{r}\right)+D\left(\frac{r_{\mathrm{f}}}{r}\right)^{2}\right] \sin \theta
$$

where

$$
A=c / 2-1-c K n, \quad B=1-c, C=-4(1 / 2+K n), \quad D=(1 / 2+K n) c, \quad c=\left(r_{\mathrm{f}} / b\right)^{2}, \quad K n=\lambda / r_{\mathrm{f}}
$$

and

$$
J=3+2 \ln c-4 c+c^{2}+\mathrm{Kn}\left(2+4 \ln c-2 c^{2}\right)
$$

where $u$ is mean velocity inside the filter, $u=u_{\infty} /(1-c), \mathrm{m} / \mathrm{s} ; u_{\infty}$ is the undisturbed approach velocity of gas (i.e., filtration velocity), $\mathrm{m} / \mathrm{s}, c$ is packing density of filter, defined as the ratio of the volume of fiber to that of Kuwabara cell, $K n$ is Knudsen number, $b$ and $r_{\mathrm{f}}$ are radius of Kuwabara cell and the fiber, respectively (see Fig. 1).

Under Cartesian coordinates, the velocity components of the Kuwabara flow field in $x$ and $y$ directions are found by using

$$
u_{x}=-\frac{r_{\mathrm{f}}^{2}}{r^{2} \sin \theta} \frac{\partial \psi}{\partial \theta}, u_{y}=\frac{r_{\mathrm{f}}^{2}}{r \sin \theta} \frac{\partial \psi}{\partial r}
$$

In the Monte Carlo simulations, the initial positions of the individual approaching particles on the "release surface" located above the filtration medium are first assigned, using a random number with uniform deviates between 0 and 1 (see Fig. 1). Once released, the particle is allowed to move in any of four directions in terms of the motion probabilities by Eq. (8) at a given $P e$, and thus its trajectory can be determined. The knowledge of the particle trajectory, in turn, determines whether or not a given particle will be deposited, and if so, the deposition position. If a particle migrates outside the simulation domain in $x$ direction, it reenters on the opposing side at the same height (i.e., periodic boundary conditions). If a particle migrates too far from the deposit layer, (beyond the "kill line"), it is discarded from the simulation and a new particle is released. This procedure is repeated until the number of the deposited particles reaches a preset value.

\section{Results and Discussion}

To investigate the effect of the above-mentioned particles transport mechanism (measured by $P e$ ) on the the resulting microstructure of dust cake, simulation runs were performed for different $P e$ ranging from 1 to 10000. The number of deposited particles is set to 5000 for $P e \geq 10$ and 2000 for $P e$ $<10$ in order to reduce computational time. In present study, for each case, at least ten independent simulation runs using different set of random numbers were performed repeatedly for obtaining meaningful the ensemble average of the results. The other simulation parameters used in the present study is as follows: the packing density $c=0.2$, the ratio of the particle diameter to fiber is set to 0.2 and the number of fibers is ten in all simulation runs.

Fig. 2 shows that the morphologies of dust cakes depending upon different $P e$ values are shown in Fig. 2. It is observed that as the $P e$ decreases, i.e., as the importance of diffusion increases, the deposition structure becomes more open and loose, and the dendric patterns begin to appear. For $P e=1$, for example, it is observed that a huge dendric structure formed on a fiber large space distribution. It is expect that such huge particles dendrite capture the subsequent approaching particles more effectively, and that the smaller particles dendrites near to it quickly become inaccessible to the approaching particles and stop growing owning to the "shadowing effect" resulting from such huge dendric structure. On the other hand, a high $P e$ values tend to give a compact deposition structure, with little porosity and few branches. 


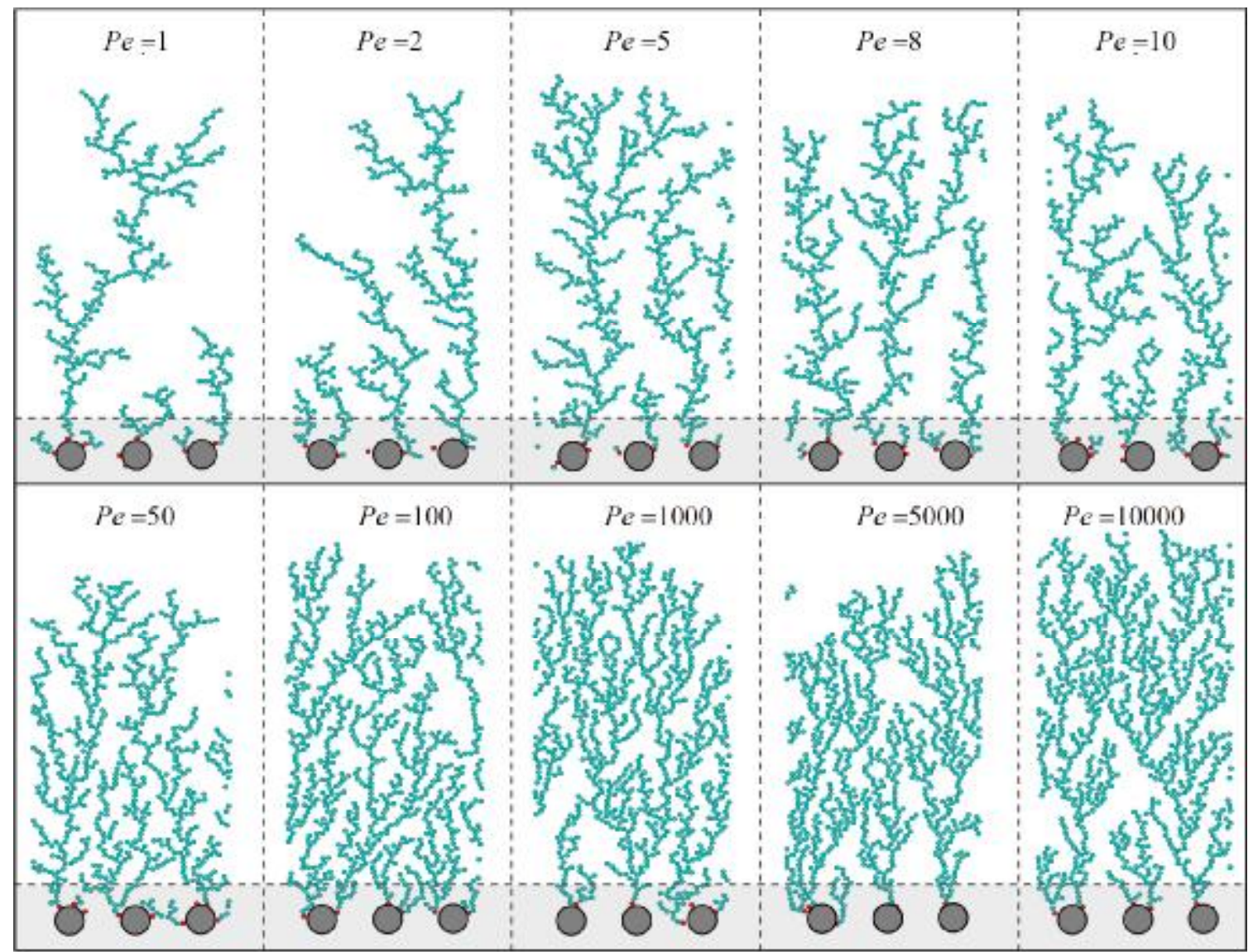

Fig. 2 Simulated morphological structures of dust cakes for different Peclet number

As mentioned above, the dynamic performance of the dust cake filtration strongly depends on cake porosity.To quantitatively investigate the microstructure of dust cakes formed on various conditions. The density profiles of cakes for different Pe are calculated and are plotted in Fig. 3 . The densities of dust cakes for lower $P e(P e<10)$, for which transport dominated by diffusion, are observed to decrease continually with height (see Fig. 2a). However, in the case of $P e \geq 10$, where the transport dominated by convection, increase to near a plateau value for intermediate heights, after an initial rapid increase density, and then decreases rapidly zero in the upper deposition areas.The initial deposition stages (thus, lower heights) are affected by the presence of the fibers. This feature of cakes density is good agree with the morphologies in Fig. 2. According to classical single fiber filtration theory, single fiber collection efficiency dereases with increasing Peclet number, which means a larger Pe will lead to a smaller number of particles directly deposited on surface of fibers. In this deposit, it is expect that the deposit near the fibers may be more prone to break off, with little support.
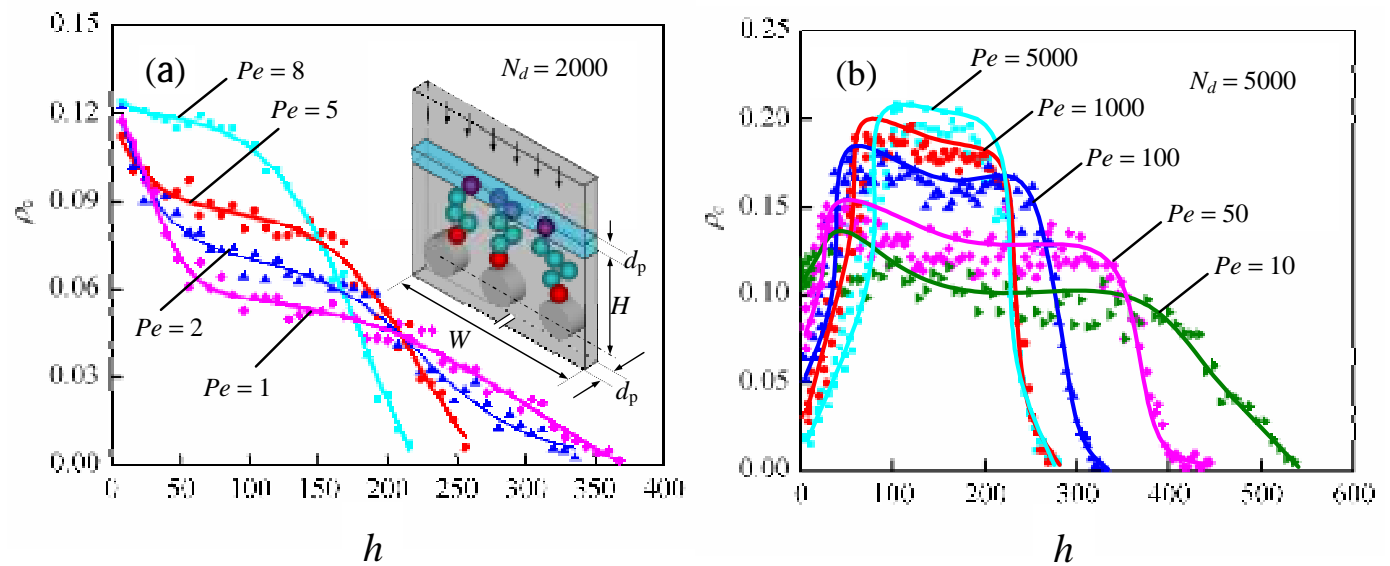

Fig. 3 Dependenc of densities on height of dust cakes for different Peclet number. $\left(N_{\mathrm{d}}\right.$ : number of deposited particles ). 
In order to further quantitatively characterize the complex structure of these dust cakes, an analysis by fractal dimension $(D)$ was carried out. The fractal dimension is a ratio providing a statistical index of complexity comparing how detail in a pattern changes with the scale at which it is measured. It has also been characterized as a measure of the space-filling capacity of a pattern that tells how a fractal scales differently from the space it is embedded in [14,15].In this study, the fractal dimension of dust cakes was calcualted from upper surface properties by plotting average height of the dust cakes formde $(\bar{h})$ versus the by numbers of particles deposited $(N)$ dust cakes [7,11]. According to the dependence $\bar{h} \propto N^{\phi}$, the fractal dimension is obtained by $D=d-d_{s}+1 / \phi$, where $d$ is the smallest Euclidean dimension of the space inwhich the fractal is embedded, and ds is dimension of the surface of nucleation sites ( $d=2$ and $d_{\mathrm{s}}=1$ in present study). For the value of each Peclet number, ten independent simulation runs were carried out to obtain meaningful average results, and the values of the fractal dimension for dust cakes under different Peclet number are summarized in Table 1. It can be found that the fractal dimensions of the dust cakes increase considerably with Peclet number. A lower Peclet number leads to a smaller fractal dimension due to particle movement is dominated by diffusion. In this work, the fractal dimension of dust cake when $P e=1$ approaches to 1.700, a value for diffusion-limited aggregation (DLA) model. For Peclet number higher than 100, the morphologies of dust cakes is very close to ballistic-limited aggreation model, with values of fractal dimension slightly less than 2 corresponding to a Euclidean object.

Table 1. simulated results for dust cake fractal dimension for various Peclet number

\begin{tabular}{cccccc}
\hline$P e=1$ & $P e=5$ & $P e=10$ & $P e=100$ & $P e=1000$ & $P e=5000$ \\
\hline 1.702 & 1.776 & 1.929 & 1.964 & 1.975 & 1.976 \\
1.705 & 1.791 & 1.921 & 1.969 & 1.971 & 1.979 \\
1.712 & 1.779 & 1.936 & 1.973 & 1.979 & 1.981 \\
1.724 & 1.781 & 1.931 & 1.965 & 1.968 & 1.977 \\
1.712 & 1.804 & 1.926 & 1.971 & 1.978 & 1.985 \\
1.707 & 1.769 & 1.919 & 1.959 & 1.971 & 1.980 \\
1.701 & 1.759 & 1.941 & 1.967 & 1.985 & 1.991 \\
1.713 & 1.761 & 1.911 & 1.949 & 1.988 & 1.997 \\
1.720 & 1.809 & 1.933 & 1.981 & 1.997 & 1.975 \\
1.710 & 1.739 & 1.945 & 1.958 & 1.968 & 1.988 \\
Average & & & & & \\
$1.711 \pm 0.050$ & $1.777 \pm 0.015$ & $1.929 \pm 0.007$ & $1.966 \pm 0.006$ & $1.978 \pm 0.007$ & $1.983 \pm 0.005$ \\
\hline
\end{tabular}

\section{Conclusions}

The growth of dust cakes from ultrafine particles in a model filter composed of an array of identical parallel fibers placed transverse to the flow have been simulated based on an off-lattic Monte Carlo method. The accurate information of flow near fiber, relating to transport behavior of particles and resulting morphologies of dust cakes, was considered. The simulation results clearly show that the particles transport mechanisms (i.e., diffusion and convection) play a major role in determining the morphology of dust cakes depending upon their relative importance to each other. A smaller Peclet number tends to result in more open and loose tree-like structure characterized by with fractal geometry. Unlike previous results on particles deposition on a simply two dimensional threadiness substrate. In this study, a dust layer near filtration medium with lower porosity can be observed in the cases of $P e \geq 10$, owning to the effect of flow field in vicinity of fibers. Whereas for a lower Peclet number $(P e<10)$, there are no observed lower porosity dust layers in the vicinity of filtration medium. 


\section{Acknowledgements}

This work was financially supported by the National Natural Science Foundation of China (51178094) and Natural Science Foundation of Guilin University of Aerospace Technology (YJ1305).

\section{References}

[1] D. Vallero, Fundamentals of air pollution, Academic press, London, 2014.

[2] S.J. Miller, D.L. Landal, Pulse-jet Baghouse Performance Improvement with Flue Gas Conditioning, EPRI, 1992, RP-3083-P

[3] D. Thomas, P. Penicot, P. Contal, et al. Clogging of fibrous filters by solid aerosol particles experimental and modelling study, Chemical Engineering Science 56 (2001) 3549-3561.

[4] E.H. Tanabe, P.M. Barros, K.B. Rodrigues, et al. Experimental investigation of deposition and removal of particles during gas filtration with various fabric filters, Separation and Purification Technology 56(2011) 187-195.

[5] D. Houi, R. Lenormand, Particle accumulation at the surface of a filter, Filtration and Separation 56 (1986) 238-241.

[6] T.A. Witten, L.M. Sander, Diffusion-limited aggregation, Physical Review 27 (1983) 5686.

[7] S. Veerapaneni, M.R. Wiesner, Particle deposition on an infinitely permeable surface: Dependence of deposit morphology on particle size, Journal of Colloid and Interface Science 162 (1994) 110-122.

[8] W. Höflinger, C. Stöcklmayer, A. Hackl, Model calculation of the compression behaviour of dust filter cakes, Filtration and Separation 31(1994) 807-806.

[9] K.J. Jeon, Y.W. Jung, A simulation study on the compression behavior of dust cakes, Powder Technology 141 (2004) 1-11.

[10] W. Huang, D.B. Hibbert, Fast fractal growth with diffusion, convection and migration by computer simulation: Effects of voltage on probability, morphology and fractal dimension of electrochemical growth in a rectangular cell, Physica A: Statistical Mechanics and its Applications 233 (1996) 888-896.

[11]P. Meakin, Diffusion-controlled deposition on fibers and surfaces, Physical Review A 27 (1983) 2616.

[12] S. Kuwabara, The forces experienced by randomly distributed parallel circular cylinders or spheres in a viscous flow at small Reynolds numbers, Journal of the Physical Society of Japan 14 (1959) 527-532.

[13] J. Pich, Pressure drop of fibrous filters at small Knudsen numbers, Annals of Occupational Hygiene 9 (1966) 23-27.

[14] K. Falconer, Fractal geometry: mathematical foundations and applications, Wiley, Chichester, 2004.

[15]D. Rodríguez-Pérez, J.L. Castillo, J.C. Antoranz, Relationship between particle deposit characteristics and the mechanism of particle arrival, Physical Review E 72(2005) 021403. 